\title{
On the functorial prolongations of fiber bundles
}

\author{
Ivan Kolár
}




\title{
ON THE FUNCTORIAL PROLONGATIONS OF FIBER BUNDLES
}

\author{
IVAN KOLÁŘ
}

\begin{abstract}
We study the prolongation of various geometric structures on a fibered manifold with respect to the Weil functor $T^{A}$ or a fiber product preserving bundle functor $F$ on the category of all fibered manifolds with $m$-dimensional bases and their morphisms with local diffeomorphisms as base maps. Special attention is paid to connections, vector bundles, principal bundles and weak principal bundles.
\end{abstract}

2000 Mathematics Subject Classification: Primary 58A20; Secondary 58A32

Keywords: Weil functor, fiber product preserving bundle functor, functorial prolongation of geometric structures, weak principal bundle

There are two important classes of geometric functors that can be characterized in terms of Weil algebras. About 1987, it was clarified that the product preserving bundle functors on the category $\mathcal{M} f$ of all manifolds and all smooth maps coincide with the Weil functors $T^{A}$, see [2] for a survey. Further, in 1999 W. Mikulski and the author, [4], analogously described the fiber product preserving bundle functors $F$ on the category $\mathcal{F} \mathcal{M}_{m}$ of fibered manifolds with $m$-dimensional bases and their morphisms with local diffeomorphisms as base maps.

In the present paper, we discuss the application of both types of functors to various geometric structures on fibered manifolds and we underline the differences between both cases. In Section 1, we summarize the basic facts about $T^{A}$ (called Case 1) and $F$ (called Case 2). The prolongation of vector bundles in both cases is discussed in Section 2. In Section 3, we consider a general connection $\Gamma$ on a fibered manifold $p: Y \rightarrow M$ and its prolongation $\mathcal{T}^{A} \Gamma$ to $T^{A} p: T^{A} Y \rightarrow T^{A} M$. Section 4 is devoted to the prolongation of $\Gamma$ to $F Y \rightarrow M$. Our presentation is systematically based on the concept of flow natural map $\psi_{Y}^{F}$ of $F$ over $Y$. In both cases, special attention is paid to linear connections.

The prolongation $\mathcal{T}^{A} \Gamma$ of a principal connection $\Gamma$ on a principal bundle $P \rightarrow M$ is discussed in Section 5. We have already pointed out, [1], that the case of $F P \rightarrow M$ is much more complicated. In Section 6, we characterize some induced connections by the properties related with our original concept of weak principal bundle, [2].

All manifolds and maps are assumed to be infinitely differentiable. Unless otherwise specified, we use the terminology and notation from the book [3].

The author was supported by GACR under the grant 201/09/0981. 


\section{TWO TYPES OF BUNDLE FUNCTORS}

We recall that a Weil algebra is a finite dimensional, commutative, associative and unital algebra of the form $A=\mathbb{R} \times N$, where $N$ is the ideal of all nilpotent elements of $A$. Since $N$ is finite dimensional, there exists an integer $r$ such that $N^{r+1}=0$. The smallest $r$ with this property is called the order of $A$. On the other hand, the dimension $w A$ of the vector space $N / N^{2}$ is the width of $A$. We say that a Weil algebra of width $k$ and order $r$ is a Weil $(k, r)$-algebra, [2].

The simpliest example of a Weil $(k, r)$-algebra is

$$
\mathbb{D}_{k}^{r}=\mathbb{R}\left[x_{1}, \ldots, x_{k}\right] /\left\langle x_{1}, \ldots, x_{k}\right\rangle^{r+1}=J_{0}^{r}\left(\mathbb{R}^{k}, \mathbb{R}\right) .
$$

For $k=r=1, \mathbb{D}_{1}^{1}=\mathbb{D}$ is the algebra of Study numbers. In [2], we deduced

Lemma 1. Every Weil $(k, r)$-algebra is a factor algebra of $\mathbb{D}_{k}^{r}$. If $\varrho, \sigma: \mathbb{D}_{k}^{r} \rightarrow A$ are two algebra epimorphisms, then there exists an algebra isomorphism $\chi: \mathbb{D}_{k}^{r} \rightarrow$ $\mathbb{D}_{k}^{r}$ such that $\varrho=\sigma \circ \chi$.

We apply the covariant approach to Weil functors, $[2,3]$.

Definition 1. Two maps $\gamma, \delta: \mathbb{R}^{k} \rightarrow M$ determine the same $A$-velocity $j^{A} \gamma=$ $j^{A} \delta$ if for every smooth function $\varphi: M \rightarrow \mathbb{R}$,

$$
\varrho\left(j_{0}^{r}(\varphi \circ \gamma)\right)=\varrho\left(j_{0}^{r}(\varphi \circ \delta)\right) .
$$

By Lemma 1, this is independent of the choice of $\varrho$.

We say that

$$
T^{A} M=\left\{j^{A} \gamma ; \gamma: \mathbb{R}^{k} \rightarrow M\right\}
$$

is the bundle of all $A$-velocities on $M$. For every smooth map $f: M \rightarrow N$, we define $T^{A} f: T^{A} M \rightarrow T^{A} N$ by

$$
T^{A} f\left(j^{A} \gamma\right)=j^{A}(f \circ \gamma)
$$

Clearly, $T^{A} \mathbb{R}=A$.

Case 1. We say that (1.2) and (1.3) represent the covariant approach to Weil bundles. The following result is a fundamental assertion, see [2] for a survey.

Theorem 1. The product preserving bundle functors on $\mathcal{M} f$ are in bijection with $T^{A}$. The natural transformations $T^{A_{1}} \rightarrow T^{A_{2}}$ are in bijection with the algebra homomorphisms $\mu: A_{1} \rightarrow A_{2}$.

We write $\mu_{M}: T^{A_{1}} M \rightarrow T^{A_{2}} M$ for the value of $\mu: A_{1} \rightarrow A_{2}$ on $M$. If $A_{i}$ is a Weil $\left(k_{i}, r_{i}\right)$-algebra, $i=1,2$, then there exists a polynomial map $\bar{\mu}: \mathbb{R}^{k_{2}} \rightarrow \mathbb{R}^{k_{1}}$ such that

$$
\mu_{M}\left(j^{A_{1}} \gamma\right)=j^{A_{2}}(\gamma \circ \bar{\mu}), \quad \gamma: \mathbb{R}^{k_{1}} \rightarrow M .
$$

The iteration $T^{A_{2}} T^{A_{1}}$ corresponds to the tensor product of $A_{1}$ and $A_{2}$. The algebra exchange homomorphism ex: $A_{1} \otimes A_{2} \rightarrow A_{2} \otimes A_{1}$ defines a natural exchange transformation $T^{A_{2}} T^{A_{1}} \rightarrow T^{A_{1}} T^{A_{2}}$. We have $T=T^{\mathbb{D}}$. 
The canonical exchange $\varkappa_{M}^{A}: T^{A} T M \rightarrow T T^{A} M$ is called flow natural. Indeed, if $F l_{t}^{X}$ is the flow of a vector field $X: M \rightarrow T M$, then

$$
\mathcal{T}^{A} X=\left.\frac{\partial}{\partial t}\right|_{0} T^{A}\left(F l_{t}^{X}\right): T^{A} M \rightarrow T T^{A} M
$$

is the flow prolongation of $X$. On the other hand, $T^{A} X: T^{A} M \rightarrow T^{A} T M$ is the functorial prolongation of $X$. One deduces easily, [2],

$$
\mathcal{T}^{A} X=\varkappa_{M}^{A} \circ T^{A} X
$$

Case 2. Consider now a bundle functor $F$ on $\mathcal{F} \mathcal{M}_{m}$ preserving fiber products. Examples are the $r$ th jet prolongation $J^{r} Y, V^{A} Y=\cup_{x \in M} T^{A}\left(Y_{x}\right), \cup_{x \in M} J_{x}^{r}\left(M, Y_{x}\right)$ and iterations.

We say $F$ is of the base order $r$ if for two $\mathcal{F} \mathcal{M}_{m}$-morphisms $\varphi, \psi: Y \rightarrow Z$ with base maps $\underline{\varphi}, \underline{\psi}: M \rightarrow N, j_{x}^{r} \underline{\varphi}=j_{x}^{r} \underline{\psi}$ and $\varphi\left|Y_{x}=\psi\right| Y_{x}$ imply $F \varphi \mid F_{x} Y=$ $F \psi \mid F_{x} Y, \bar{x} \in \bar{M}$.

Write $\mathcal{M} f_{m}$ for the category of $m$-dimensional manifolds and their local diffeomorphisms. The construction of product fibered manifolds defines injection $\iota: \mathcal{M} f_{m} \times$ $\mathcal{M} f \rightarrow \mathcal{F} \mathcal{M}_{m}, \iota(M, N)=(M \times N \rightarrow M), \iota\left(f_{1}, f_{2}\right)=f_{1} \times f_{2}, f_{1}: M \rightarrow M^{\prime}$, $f_{2}: N \rightarrow N^{\prime}$.

W. Mikulski and the author deduced, [4], that the bundle functors $\Phi=F \circ \iota$ on $\mathcal{M} f_{m} \times \mathcal{M} f$ are in bijection with the pairs $(A, H)$, where $A$ is a Weil algebra and $H: G_{m}^{r} \rightarrow$ Aut $A$ is a group homomorphism of the $r$ th jet group in dimension $m$ into the group of all algebra automorphisms of $A$. Since $H(g): A \rightarrow A$ is an algebra automorphism for every $g \in G_{m}^{r}$, we have the induced action $H_{N}(g)=$ $H(g)_{N}: T^{A} N \rightarrow T^{A} N$ of $G_{m}^{r}$ on $T^{A} N$. Then $\Phi(M, N)$ is the associated fiber bundle $P^{r} M\left[T^{A} N\right]$. For a local diffeomorphism $f_{1}: M \rightarrow M^{\prime}$ and a smooth map $f_{2}: N \rightarrow N^{\prime}$,

$$
\Phi\left(f_{1}, f_{2}\right)=P^{r} f_{1}\left[T^{A} f_{2}\right]: \Phi(M, N) \rightarrow \Phi\left(M^{\prime}, N^{\prime}\right),
$$

where $P^{r} f_{1}: P^{r} M \rightarrow P^{r} M^{\prime}$ is the induced local isomorphism of principal fiber bundles and $T^{A} f_{2}: T^{A} N \rightarrow T^{A} N^{\prime}$ is a $G_{m}^{r}$-equivariant map, [2,4].

Then the functor $F$ is determined by adding an equivariant algebra homomorphism $t: \mathbb{D}_{m}^{r} \rightarrow A$, where Aut $\mathbb{D}_{m}^{r}=G_{m}^{r}$. We have

$$
F Y=\left\{\{u, Z\} \in P^{r} M\left[T^{A} Y\right], t_{M} u=T^{A} p(Z), u \in P^{r} M, Z \in T^{A} Y\right\},
$$

where $t_{M}: T_{m}^{r} M \rightarrow T^{A} M$ and $P^{r} M \subset T_{m}^{r} M$. For an $\mathcal{F} \mathcal{M}_{m}$-morphism $f: Y \rightarrow Y^{\prime}$ over $f: M \rightarrow M^{\prime}, F f$ is the restriction of $\Phi(f, f)$ to $F Y$. In the product case $Y=\bar{M} \times N$, we have

$$
F(M \times N)=P^{r} M\left[T^{A} N\right] .
$$

If we consider another fibered manifold $Y^{\prime} \rightarrow M$ over $M$ and $\underline{f}=\mathrm{id}_{M}$, we have

$$
F f(\{u, Z\})=\left\{u, T^{A} f(Z)\right\} .
$$


Formally, $t$ induces a natural map

$$
\tilde{t}_{Y}: J^{r} Y \rightarrow F Y, \quad\{u, Z\} \mapsto\left\{u, t_{Y}(Z)\right\} .
$$

Geometrically, we interpret a section $s: M \rightarrow Y$ as a morphism $\tilde{s}: \widetilde{M} \rightarrow Y$, where $\widetilde{M}=(M \stackrel{\text { id }}{\longrightarrow} M)$ is the "doubled" manifold. Then $F \tilde{s}$ is identified with $j^{r} s$ and $\tilde{t}_{Y}\left(j_{x}^{r} s\right)=(F \tilde{s})(x)$.

\section{Prolongation of Vector Bundles}

Consider a vector bundle $p: E \rightarrow M$. The vector addition in $E$ and the multiplication of vectors by real numbers are two maps

$$
a: E \times_{M} E \rightarrow E, \quad m: \mathbb{R} \times E \rightarrow E .
$$

Applying $T^{A}$, we obtain

$$
T^{A} a: T^{A} E \times{ }_{T^{A} M} T^{A} E \rightarrow T^{A} E, \quad T^{A} m: A \times T^{A} E \rightarrow T^{A} E .
$$

If we use $\mathbb{R} \subset A$ and express all the basic properties of a vector bundle in terms of diagrams, we prove

Proposition 1. $T^{A} p: T^{A} E \rightarrow T^{A} M$ is also a vector bundle.

However, our concept of $A$-velocity offers a more geometric proof, that is, one of jet-like character. We have

$$
T^{A} E=\left\{j^{A} \gamma, \gamma: \mathbb{R}^{k} \rightarrow E\right\} .
$$

For $c \in \mathbb{R}$, we define $c j^{A} \gamma(\tau)=j^{A}(c \gamma(\tau)), \tau \in \mathbb{R}^{k}$. If $p \circ \gamma_{1}=p \circ \gamma_{2}$, we set

$$
j^{A}\left(\gamma_{1}(\tau)\right)+j^{A}\left(\gamma_{2}(\tau)\right)=j^{A}\left(\gamma_{1}(\tau)+\gamma_{2}(\tau)\right)
$$

with addition in the individual fibers of $E$. Then we verify easily that $T^{A} E$ is a vector bundle.

We underline that the map $T^{A} m: A \times T^{A} E \rightarrow T^{A} E$ introduces an action of the Weil algebra $A$ on $T^{A} E$, which is important in several applications, [2].

In the case of $F=(A, H, t)$, we can apply $F$ directly to the first formula in (2.1). This yields

$$
F a: F E \times_{M} F E \rightarrow F E
$$

with the fiber product over $M$, not over $T^{A} M$ as in (2.2). Further, $F$ can be applied to fibered manifolds only. Hence, we have to rewrite the second formula from (2.1) as

$$
m:(M \times \mathbb{R}) \times_{M} E \rightarrow E
$$

Using (1.8), we find

$$
F m: P^{r} M[A] \times_{M} F E \rightarrow F E
$$

with the action of $G_{m}^{r}$ on $A=\mathbb{R} \times N$ determined by $H$. But $\mathbb{R}$ is invariant with respect to every $H(g)$, so that $M \times \mathbb{R} \subset P^{r} M[A]$. Restricting (2.5) to this subspace, 
we obtain the multiplication by real scalars on $F E$. A direct discussion of all the related diagrams imply

Proposition 2. $F E \rightarrow M$ is also a vector bundle.

Even here, it is more geometric to apply the jet like approach based on (1.7). Two $V_{1}, V_{2} \in F_{x} E$ can be written as

$$
V_{1}=\left\{u, Z_{1}\right\}, \quad V_{2}=\left\{u, Z_{2}\right\}, \quad Z_{1}, Z_{2} \in T^{A} E
$$

with the same $u \in P_{x}^{r} M$. Then $T^{A} p\left(Z_{1}\right)=t_{M} u=T^{A} p\left(Z_{2}\right)$ imply that $Z_{1}$ and $Z_{2}$ lie in the same fiber of $T^{A} E$ over $t_{M} u \in T^{A} M$. But $T^{A} E \rightarrow T^{A} M$ is a vector bundle and $t$ is invariant. By (1.4), $V_{1}+V_{2}=\left\{u, Z_{1}+Z_{2}\right\}$ is defined in an intrinsic way. This leads directly to another proof of our assertion.

\section{INDUCED CONNECTIONS ON $T^{A} Y \rightarrow T^{A} M$}

Consider a general connection $\Gamma$ on $Y$ as a lifting map $\Gamma: Y \times_{M} T M \rightarrow T Y$. In [3], we constructed the induced general connection $\mathcal{T}^{A} \Gamma$ on $T^{A} p: T^{A} Y \rightarrow T^{A} M$ by a commutative diagram

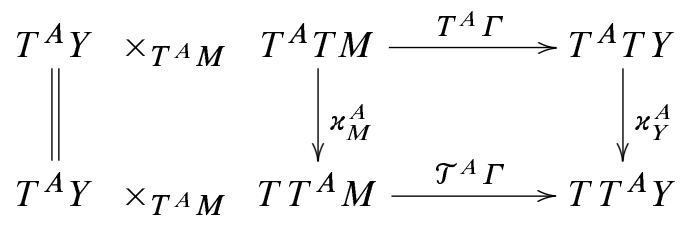

Proposition 3. Let $\Gamma$ be a linear connection on the vector bundle $p: E \rightarrow M$. Then $\mathcal{T}^{A} \Gamma$ is also linear.

Proof. Linearity of $\Gamma$ means

$$
\Gamma\left(u_{1}, X\right)+\Gamma\left(u_{2}, X\right)=\Gamma\left(u_{1}+u_{2}, X\right), \quad u_{1}, u_{2} \in E_{x}, X \in T_{x} M .
$$

We have

$$
\mathcal{T}^{A} \Gamma: T^{A} E \times{ }_{T^{A} M} T T^{A} M \rightarrow T T^{A} E
$$

and

$$
\begin{gathered}
\mathcal{T}^{A} \Gamma\left(j^{A} \gamma, j^{A} \xi\right)=j^{A} \Gamma(\gamma, \xi), \\
\gamma: \mathbb{R}^{k} \rightarrow E, \quad \xi: \mathbb{R}^{k} \rightarrow T M, \quad p \circ \gamma=\pi \circ \xi, \quad \pi: T M \rightarrow M .
\end{gathered}
$$

Then

$$
\begin{aligned}
& \mathcal{T}^{A} \Gamma\left(j^{A} \gamma_{1}+j^{A} \gamma_{2}, j^{A} \xi\right)=j^{A} \Gamma\left(\gamma_{1}(\tau)+\gamma_{2}(\tau), \xi(\tau)\right)= \\
& \quad=j^{A} \Gamma\left(\gamma_{1}, \xi\right)+j^{A} \Gamma\left(\gamma_{2}, \xi\right)=\mathcal{T}^{A} \Gamma\left(j^{A} \gamma_{1}, j^{A} \xi\right)+\mathcal{T}^{A} \Gamma\left(j^{A} \gamma_{2}, j^{A} \xi\right) .
\end{aligned}
$$

This completes the proof. 
Example 1. We present the case $T^{A}=T$ in coordinates. Let $x^{i}, y^{p}$ be some fiber coordinates on $Y$ and

$$
d y^{p}=F_{i}^{p}(x, y) d x^{i}
$$

be the equations of a general connection $\Gamma$. If $x_{1}^{i}, y_{1}^{p}$ are the additional coordinates on $T Y$, then the additional equations of $\mathcal{T} \Gamma$ are, see [2],

$$
d y_{1}^{p}=\left(\frac{\partial F_{i}^{p}}{\partial x^{j}} x_{1}^{j}+\frac{\partial F_{i}^{p}}{\partial y^{q}} y_{1}^{q}\right) d x^{i}+F_{i}^{p} d x_{1}^{i} .
$$

In the linear case, we have

$$
\begin{gathered}
d y^{p}=\Gamma_{q i}^{p}(x) y^{q} d x^{i}, \\
d y_{1}^{p}=\left(\frac{\partial \Gamma_{q i}^{p}}{\partial x^{j}} y^{q} x_{1}^{j}+\Gamma_{q i}^{p} y_{1}^{q}\right) d x^{i}+\Gamma_{q i}^{p} y^{q} d x_{1}^{i},
\end{gathered}
$$

that is linear in $y^{p}$ and $y_{1}^{p}$.

\section{INDUCED CONNECTIONS ON $F Y \rightarrow M$}

It was clarified in several concrete problems that if $F$ is of base order $r$, we need an auxiliary linear splitting $\Lambda: T M \rightarrow J^{r} T M$ to construct an induced connection $\mathcal{F}(\Gamma, \Lambda): F Y \times_{M} T M \rightarrow T F Y,[3]$. Consider a vector field $\xi: M \rightarrow T M$ and its $\Gamma$-lift $\Gamma \xi: Y \rightarrow T Y$. The flow prolongation $\mathcal{F}(\Gamma \xi): F Y \rightarrow T F Y$ depends on $j^{r} \xi$ only. This yields $\mathscr{F} \Gamma: F Y \times{ }_{M} J^{r} T M \rightarrow T F Y$ linear in $J^{r} T M$. Then $\mathscr{F}(\Gamma, \Lambda)=\mathscr{F} \Gamma \circ\left(\operatorname{id}_{F Y}, \Lambda\right)$.

It is useful to describe this construction by using the flow natural map $\psi_{Y}^{F}$. In Section 1, we constructed $t_{T M}: J^{r} T M \rightarrow F T M$. Consider a projectable vector field $\eta: Y \rightarrow T Y$ over $\xi: M \rightarrow T M$. If we interpret $\eta$ as an $\mathcal{F} \mathcal{M}$-morphism $\eta: Y \rightarrow T Y$ over id $_{M}$, we can construct $F \eta: F Y \rightarrow F T Y$. By [2], there is a unique map

$$
\psi_{Y}^{F}: F T Y \times_{F T M} J^{r} T M \rightarrow T F Y
$$

satisfying

$$
\mathscr{F} \eta=\psi_{Y}^{F}\left(F \eta, j^{r} \xi\right)
$$

for every $\eta$. This map is linear in $J^{r} T M$. Hence

$$
\mathscr{F}(\Gamma, \Lambda)=\psi_{Y}^{F}\left(F(\Gamma \xi), j^{r} \xi\right) \circ \Lambda=\mathscr{F} \Gamma \circ\left(\operatorname{id}_{F Y}, \Lambda\right) .
$$

In the case of a vector bundle $E, \psi_{E}^{F}$ is linear also in $F T E$, [2]. This implies directly

Proposition 4. If $\Gamma$ is linear, then $\mathcal{F}(\Gamma, \Lambda)$ is linear for every $\Lambda$. 


\section{The PRINCIPAL BUNDLE $T^{A} P \rightarrow T^{A} M$}

Consider a principal $G$-bundle $p: P \rightarrow M$ and write $u \cdot g, u \in P, g \in G$ for the action of $G$ on $P$. We shall use the same symbol for the induced action of $T^{A} G$ on $T^{A} p: T^{A} P \rightarrow T^{A} M$

$$
j^{A} u(\tau) \cdot j^{A} g(\tau)=j^{A}(u(\tau) \cdot g(t)), \quad \tau \in \mathbb{R}^{k} .
$$

This is a right action. If $j^{A} u_{1}(\tau)$ and $j^{A} u_{2}(\tau)$ satisfy $p \circ u_{1}=p \circ u_{2}$, then the relation

$$
u_{2}(\tau)=u_{1}(\tau) \cdot g(\tau)
$$

implies that $T^{A} P \rightarrow T^{A} M$ is a principal $T^{A} G$-bundle.

The induced action of $G$ on $T P$ is

$$
\left(\left.\frac{\partial}{\partial t}\right|_{0} v(t)\right) \cdot g=\left.\frac{\partial}{\partial t}\right|_{0}(v(t) \cdot g), \quad v: \mathbb{R} \rightarrow P .
$$

A principal connection $\Gamma: P \times_{M} T M \rightarrow T P$ is characterized by

$$
\Gamma\left(u,\left.\frac{\partial}{\partial t}\right|_{0} \gamma(t)\right) \cdot g=\Gamma\left(u \cdot g,\left.\frac{\partial}{\partial t}\right|_{0} \gamma(t)\right), \quad \gamma: \mathbb{R} \rightarrow M .
$$

The induced action of $T^{A} G$ on $T T^{A} P$ is

$$
\left(\left.j^{A} \frac{\partial}{\partial t}\right|_{0} u(\tau, t)\right) \cdot j^{A} g(\tau)=\left.\frac{\partial}{\partial t}\right|_{0} j^{A}(u(\tau, t) \cdot g(\tau)),
$$

$u: \mathbb{R}^{k} \times \mathbb{R} \rightarrow P, g: \mathbb{R}^{k} \rightarrow G$

Proposition 5. If $\Gamma$ is a principal connection on $P \rightarrow M$, then $\mathcal{T}^{A} \Gamma$ is a principal connection on $T^{A} P \rightarrow T^{A} M$.

Proof. For $u: \mathbb{R}^{k} \rightarrow P, g: \mathbb{R}^{k} \rightarrow G, \gamma: \mathbb{R}^{k} \times \mathbb{R} \rightarrow M$, we obtain by (5.3) and (5.4)

$$
\begin{aligned}
\mathcal{T}^{A} \Gamma\left(j^{A}(u(\tau)) \cdot j^{A} g(\tau),\left.\frac{\partial}{\partial t}\right|_{0} j^{A} \gamma(\tau, t)\right)= & \\
& =\mathcal{T}^{A} \Gamma\left(j^{A}(u(\tau)),\left.\frac{\partial}{\partial t}\right|_{0} j^{A} \gamma(\tau, t)\right) \cdot j^{A} g(\tau) .
\end{aligned}
$$

This completes the proof of the proposition.

In the case of a bundle $P[S]$ associated to $P$ with respect to a left action $G \times S \rightarrow$ $S$, every principal connection $\Gamma$ on $P$ induces a general connection $\Gamma_{S}$ on $P[S]$. If $\Gamma(u, X)=\left.\frac{\partial}{\partial t}\right|_{0} \gamma(t), \gamma: \mathbb{R} \rightarrow P$, then

$$
\Gamma_{S}(\{u, a\}, X\}=\left.\frac{\partial}{\partial t}\right|_{0}\{\gamma(t), a\}, \quad a \in S .
$$

Consider the induced action $T^{A} G \times T^{A} S \rightarrow T^{A} S$. Analogously to Proposition 5, we deduce 
Proposition 6. We have $\mathcal{T}^{A}\left(\Gamma_{S}\right)=\left(\mathcal{T}^{A} \Gamma\right)_{T^{A} S}$.

\section{WEAK PRINCIPAL BUNDLES}

It is well known that the $r$ th jet prolongation $J^{r} P \rightarrow M$ of a principal $G$-bundle $P \rightarrow M$ is not a principal bundle, [2]. When investigating the functor $F=(A, H, t)$, we realized that some general phenomena are reflected in the concept of the weak principal bundle, [2]. Let $K$ be a Lie group.

Definition 2. A fibered manifold $p: C \rightarrow M$ is called a group bundle of type $K$ if each fiber is a Lie group and for every $x \in M$ there exists a neighbourhood $U$ such that $p^{-1}(U) \approx U \times K$.

The group compositions form a base preserving morphism $v: C \times_{M} C \rightarrow C$.

Lemma 2. $F v: F C \times_{M} F C \rightarrow F C$ is a group bundle of type $T^{A} K$.

Proof. By locality, we may assume $C=M \times K$. Write $x: K \times K \rightarrow K$ for the group composition in $K$. Consider $c_{1}, c_{2} \in F_{x} C, c_{1}=\left\{u, Z_{1}\right\}, c_{2}=\left\{u, Z_{2}\right\}$, $u \in P_{x}^{r} M, Z_{1}, Z_{2} \in T^{A} K$. Using (1.9), we obtain

$$
F \varkappa\left(\left\{u, Z_{1}\right\},\left\{u, Z_{2}\right\}\right)=\left\{u, T^{A} \varkappa\left(Z_{1}, Z_{2}\right)\right\} .
$$

The lemma is now proved.

Definition 3. A fibered manifold $Q \rightarrow M$ is called a weak principal bundle with structure group bundle $C \rightarrow M$ if we are given a base-preserving morphism $\varrho: Q \times_{M}$ $C \rightarrow Q$ such that each group $C_{x}$ acts simply transitively on the right on $Q_{x}$.

Clearly, the principal bundle is a weak principal bundle, the group bundle of which is the product $M \times K$.

Example 2. We have $F(M \times G)=P^{r} M\left[T^{A} G\right]$. This is a group bundle of type $T^{A} G$. Applying $F$ to $\varrho: P \times_{M}(M \times G) \rightarrow P$, we obtain $F \varrho: F P \times_{M} P^{r} M\left[T^{A} G\right] \rightarrow$ $F P$. This defines a weak principal bundle structure on $F P$ for every principal bundle $P$.

Proposition 7. For a weak principal bundle $\varrho: Q \times{ }_{M} C \rightarrow Q, F \varrho: F Q \times{ }_{M} F C \rightarrow$ $F Q$ is also a weak principal bundle.

Proof. By locality, the problem reduces to Example 2.

Definition 4. A connection $\Delta: C \times_{M} T M \rightarrow T C$ is called a group connection if $\Delta\left(c_{i}, X\right)=\left.\frac{\partial}{\partial t}\right|_{0} u_{i}(t), i=1,2$, imply $\Delta\left(\varkappa\left(c_{i}, c_{2}\right), X\right)=\left.\frac{\partial}{\partial t}\right|_{0} \varkappa\left(u_{1}(t), u_{2}(t)\right)$ for every $c_{1}, c_{2} \in C_{x}, X \in T_{x} M$.

Definition 5. A connection $\Gamma: Q \times_{M} T M \rightarrow T Q$ is called weak principal with respect to a group connection $\Delta: C \times_{M} T M \rightarrow T C$ if $\Gamma(u, X)=\left.\frac{\partial}{\partial t}\right|_{0} u(t), \Delta(c, X)=$ $\left.\frac{\partial}{\partial t}\right|_{0} c(t)$ implies

$$
\Gamma(\varrho(u(0), c(0)), X)=\left.\frac{\partial}{\partial t}\right|_{0} \varrho(u(t), c(t)), u: \mathbb{R} \rightarrow Q, c: \mathbb{R} \rightarrow C .
$$


Clearly, if $C=M \times K$ is the product bundle, a weak principal connection $\Gamma$ on $Q$ with respect to the product connection on $C$ is principal.

Using the ideas of Section 4, one deduces directly

Proposition 8. If $\Gamma$ is a weak principal connection on $Q$ with respect to a group connection $\Delta$ on $C$, then $\mathcal{F}(\Gamma, \Lambda)$ is weak principal with respect to $\mathcal{F}(\Delta, \Lambda)$ for every $\Lambda$.

Remark. In the theory of prolongation of geometric object fields, one modifies the weak principal bundle $J^{r} P$ into a principal bundle by constructing $W^{r} P=$ $J^{r} P \times_{M} P^{r} M$, [3]. In [1], A. Cabras and the author have described a general procedure of this type.

\section{REFERENCES}

[1] A. Cabras and I. Kolár, "On the functorial prolongations of principal bundles," Comment. Math. Univ. Carol., vol. 47, pp. 719-731, 2006.

[2] I. Koláŕ, "Weil bundles as generalized jet spaces," in Handbook of Global Analysis. Elsevier, 2008, pp. 625-664.

[3] I. Koláŕ, P. W. Michor, and J. Slovák, Natural Operations in Differential Geometry. Springer Verlag, 1993.

[4] I. Koláŕ and W. M. Mikulski, "On the fiber product preserving bundle functors," Differential Geom. Appl., vol. 11, pp. 105-115, 1999.

Author's address

Ivan Koláŕr

Masaryk University, Institute of Mathematics and Statistics, Kotlářská 2, 61137 Brno, Czech Republic

E-mail address: kolaremath.muni.cz 